\title{
Author Index Vol. 24, 1996
}

\section{Abbott, R. 56}

Adelson, P.D. 139

Albright, A.L. 8, 126, 185,252

Albright, L. 167

Allen, J.C. 98,167

Arai, H. 1

Armstrong, D. 306

Arnold,W. 92

Aronin, P. 167

Atan, M. 285

Azzarelli, B. 160

Backstrom, J.W. 61 Barkovich, A.J. 9,242 Baumann, G.S. 193 Bell, W.O. 134 Biglan, A.W. 202 Black, P. 74 Block,|S.M. 134 Boyett, J.M. 167,185 Brand,|W.N. 178 Burger,|P.C. 35

Caldemeyer, K.S. 160 Carroll,,R. 74 Cavenee, W.K. 41 Chambers, G.K. 92 Cheek,|W. 306 Cherlow, J. 167 Chintagumpala, M.M. 306 Christian, C. 292 Ciricillo, S.F. 193 Cochrane, D.D. 92,277 Conry, J. 223 Constantini, S. 24

D’Alise, M.D. 151 Demirtas, E. 155 Deutsch, M. 167 Dhillon, M.K. 285 Dias, M.S. 61,69 Domingo, Z. 111 Drake, J.M. 55 Duffner, P. 167 Duhaime, A.-C. 292 Dunkel, IJJ. 263

Edwards, M.S.B. 9, 193, 314 Epstein, F.J. 8,24 Ersahin, Y. 155

Falkner, L.D. 323 Finlay, J.L. 167,185,263

Fischbein, N.J. 9 Fisher, R.S. 79 Fletcher, D.F. 229 Flickinger, J.C. 139 Foreman, N.K. 119 Freeman, C.R. 103 Freidman, H. 167 Friedman, A.D. 50 Furnari, F.B. 41

Gayek, RJ. 79 Geohas, J. 178 Gerszten, P.C. 139 Glenn, O.A. 242 Glowacka, D. 74 Grant, J.A. 272,275 Gutin, P.H. 193,314

Hari, J.K. 160 Hoffman, H.J. 256 Hovda, D.A. 79 Humphreys, R.P. 138

Irwin, B. 92

Jacobson, E.E. 229 Jallo, G.I. 56 Johnson, D.L. 223 Johnston, I.H. 229

Kaplan, A.M. 185 Katayama, K. 52 Klein, D.M. 61,69 Kondziolka, D. 139 Krischer, J. 167 Kun, L. 167 Kurata, H. 52

Lamb, S.A. 314 Lamborn, K.R. 314 Langston, J. 167 Larson,|D.A. 193,314 Laurent, J. 306 Li, H. 185 Li, V. 126 Linggood, R. 167 Losken, H.W. 202 Love, S. 119 Lucke, J.F. 237 Lunsford, L.D. 139 Lye, M.-S. 285

McDermott,M.W. 193 McLone, D.G. 275 Mahoney, D.H.,Jr. 306 Malec, M.K. 314 Mapstone, T.B. 8 Marymont, M.H. 178 Miki, S. 306 Mittal, B.B. 178 Miyajima, M. 1 Mochizuki, M. 52 Morgan, M.K. 229 Morota, N. 56 Moss, E. 292 Mulhern, R. 167 Mutluer, S. 155

Nagib, M.G. 299 Nakamura, H. 52 Nelson, L.H. 134 Nelson, T. 306

O’Donnell, R. 223 O’Fallon, M.T. 299 O’Malley, B. 263 Oi, S. 52

Olmstead, C.E. 79 Ong, L.-c. 285

\section{Packer, R.J. 167,185,211,217}

Pang, D. 126

Park, E. 193

Peacock, W.J. 79

Peter, J. Ill

Petti, P.L. 193

Phillips, T.L. 314

Pittman, T. 50

Pollack, I.F. 202

Prados, M.D. 9,314

Reigel, D.H. 237 Rhoney, J.C. 134 Rorke, L.B. 167,185 Rotenstein, D. 237 Russo, Cl 9,314

Sarwark, J.F. 272 Sato, K. 1 Sauntry, S. 272 Sawa, H. 52 Saydam, S. 155 Scharfen, CO. 314 Sclabassi, R. 126 Seidl, T. 292

Selladurai, B.M. 285 Shapiro, R.H. 5 Shiminski-Maher, T. 267 Siffert, J. 98 Smith, J.L. 323 Smith,VV. 193 Sneed, P.K. 193,314 Song, H.-R. 272 Stalpers, L.J.A. 193 Stanley, P. 167 Stehbens, J.A. 167 Steinberg, G.K. 145 Strauss, L. 178 Su Huang, H.-J. 41 Swift, D.M. 151

Tamaki, N. 52 Thiessen, P.N. 92 Thomas, P.R.M. 167 Thome, R. 119 Timmons, C.F. 151 Tomita, T. 178 Tosun, A.R. 155

Uetani, Y. 52 
Verhey, LJJ. 193 Vernon, D.D. 323 Vietti, T. 167 Voss, B. 314

Wald, S.L. 5

Walker, M.L. 279, 323

Wang, M.Y. 145

Wara, W.M. 9,185,193,314

Weaver,K.A. 314

Wehby-Grant, M.C. 79

Williams, D. 50

Willis,,B.K. 323

Wilson, J.T. 5

Woo, S.Y. 306

Yokoyama, N. 52

Zimmerman, R.A. 185

\section{KAIU;EII}

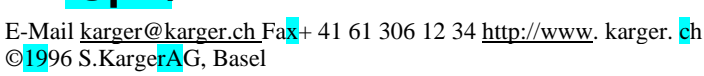


